\title{
Influence of Jets on Anisotropic Flow in Relativistic Heavy Ion Collisions
}

\author{
Larissa Bravina* \\ Department of Physics, University of Oslo, Oslo, Norway \\ E-mail: larissa.bravina@fys.uio.no

\section{Bengt Henrik Brusheim Johansson} \\ Department of Physics, University of Oslo, Oslo, Norway \\ E-mail: bhjohanestudent.matnat.uio.no
}

\section{Gyulnara Eyyubova}

Department of Physics, University of Oslo, Oslo, Norway and Skobeltzyn Institute of Nuclear Physics, Moscow State University, Moscow, Russia E-mail: Gyulnara.Eyyubova@cern.ch

\section{Evgeny Zabrodin}

Department of Physics, University of Oslo, Oslo, Norway and Skobeltzyn Institute of Nuclear Physics, Moscow State University, Moscow, Russia E-mail: eugen.zabrodinefys.uio.no

The interplay between soft processes, represented by parametrized hydrodynamics, and jets is studied for ultrarelativistic heavy-ion collisions at RHIC and LHC energies. For simulations of the collisions the HYDJET++ model is employed. It is shown that jets can be responsible for the following features of particle anisotropic flow: (i) drop of the elliptic flow at $p_{T} \geq 2.5 \mathrm{GeV} / c$; (ii) rise of the ratio $R=v_{4} / v_{2}^{2}$ compared to theoretical estimate $R=0.5$; (iii) rise of high- $p_{T}$ tail of this ratio; (iv) violation of the number-of-constituent-quark (NCQ) scaling at LHC.

Xth Quark Confinement and the Hadron Spectrum,

October 8-12, 2012

TUM Campus Garching, Munich, Germany

\footnotetext{
* Speaker.
} 


\section{Introduction}

The experiments on ultrarelativistic heavy ion collisions are of great importance, because they offer a unique opportunity to study the nuclear phase diagram at high temperatures and densities [1]. The matter under such extreme conditions probably has existed in the early Universe within the first few $\mathrm{fm} / \mathrm{c}$ 's after the Big Bang. Nowadays scientists are intensively investigating the properties of the Little Big Bang in the laboratory in order to search for a new state of matter, quark-gluon plasma (QGP) [2], predicted by quantum chromodynamics (QCD).

Collective anisotropic flow of particles, produced in heavy-ion collisions at (ultra)relativistic energies, is a powerful tool to study properties of hot and dense nuclear matter. Various model simulations show that the flow is very sensitive to changing of the equation-of-state (EOS) of the substance during the system evolution [1]. Therefore, it might carry fingerprints of the quark-gluon plasma. Nowadays the flow is studied in terms of Fourier series [3, 4]

$$
E \frac{d^{3} N}{d^{3} p}=\frac{d^{2} N}{2 \pi d p_{t} d y}\left[1+2 \sum_{n=1}^{\infty} v_{n} \cos (n \phi)\right],
$$

where the coefficients $v_{n}$ represent the harmonics of the anisotropic flow. Of particular interest for our study are the first two even harmonics, dubbed elliptic flow, $v_{2}$, and hexadecapole flow, $v_{4}$.

Briefly, the status of the problem is as follows. It was claimed in [5] that the $v_{4}$ could carry important information concerning the physics of heavy-ion collisions. However, under the general hydrodynamic treatment of the flow development, the hexadecapole flow was shown [6] to be fully determined by the elliptic flow, namely, $v_{4}=0.5 v_{2}^{2}$, thus playing a marginal role as independent source of physical information. Experimental study of the ratio $R=v 4 / v_{2}^{2}$ at RHIC energy revealed that the theoretical estimates are exceeded at least by factor $2[7,8]$. This ratio was found to be close to unity and weakly dependent on centrality of collisions and transverse momenta of identified hadrons. To resolve the obvious ambiguity between the theory and the experiment the event-byevent fluctuations of particles in the reaction plane were invoked. As mentioned in [9], the ratio $v 4 / v_{2}^{2}$ is estimated in the experiment by averaging of both elliptic and hexadecapole flow over all collisions and not on event-by-event basis. In this case $R_{\exp }$ should acquire an extra-multiplier varying slightly from 1.56 for semicentral to 1.38 for semiperipheral collisions [10] and leading to increase of $R$ despite the fact that $v 4 / v_{2}^{2}=0.5$ in every single heavy-ion collision. Finally, the LHC results favor further overall increase of the ratio and, particularly, formation of instantly rising high$p_{T}$ tail of $R$. Such a behavior cannot be reproduced in conventional hydrodynamic models relying on both ideal and viscous hydrodynamics accompanied by Glauber or Color Glass Condensate initial conditions [10].

Another important observation at LHC is the violation of the number-of-constituent-quark (NCQ) scaling of the elliptic flow [11]. The NCQ scaling was first observed at RHIC [12, 13]. It should definitely hold at LHC provided the elliptic flow is formed at partonic level.

We would like to mention here that the formation of anisotropic flow is studied up to now merely within the framework of hydrodynamics. The interplay between the soft processes (hydro) and hard processes (jets) is somehow overlooked. Therefore, for our present study we employ the HYDJET++ model containing the treatment of both soft and hard processes. Its main features are given in Sect. 2. 


\section{The HYDJET++ model}

The HYDJET++ (HYDrodynamics with JETs) event generator [14] provides fast simulation of hadronic spectra in both central and non-central heavy-ion collisions at ultra-relativistic energies. It is a marriage between the FASTMC event generator [15], that utilizes the parametrized ideal hydrodynamics to simulate the spectra of hadrons with transverse momenta $p_{T} \leq 2 \mathrm{GeV} / c$, and the HYDJET model [16], that is focused on jets traversing dense and hot partonic substance. Generation of hadrons in both "soft" and "hard" parts of the HYDJET++ proceeds independently.

Realization of fast algorithm enabling the quick generation of soft hadrons in the FASTMC is as follows. The Glauber model of independent inelastic collisions of nucleons is used to determine the mean number of interacting nucleons $N_{\text {part }}$ at certain impact parameter $b$. When the $N_{\text {part }}$ is known, the program estimates the effective volume $V_{e f f}$ of the fireball. After that the mean multiplicity of secondary hadrons produced either on Bjorken-like or Hubble-like freeze-out hypersurface is calculated. The FASTMC contains options for both separate and simultaneous treatment of chemical and thermal freeze-out of particles. Spatial and momentum anisotropies are used to parametrize elliptic flow of produced hadrons. The attractive feature of the model is a very extensive table of ca. 360 meson and baryon resonances taken from the SHARE model [17]. Their decay modes and branching ratios are implemented as well. The free parameters of the model are tuned to reproduce simultaneously hadron yields and ratios, femtoscopy correlations and elliptic flow in heavy-ion collisions at RHIC and at LHC energies.

The HYDJET model propagates hard partons through hot and dense medium, e.g. quark-gluon plasma. Every A+A collision is subdivided into $N N$ collisions. Simulation of an individual $N N$ collision includes the following steps: (i) generation by means of PYTHIA [18] of production vertexes and individual parton spectra for a given impact parameter, (ii) calculation of gluon radiation and collisional energy losses for a parton traversing dense and hot medium, (iii) estimation of cold nuclear matter effects, such as quark and gluon shadowing, and finally, (iv) hadronization of both quarks and gluons according to prescriptions of Lund model [19]. The mean number of multi-jets generated in a single $\mathrm{A}+\mathrm{A}$ collision at a given impact parameter is a product of the number of binary collisions experienced by all partons and the integral cross section of the hard process in $N N$ collision with the minimum transverse momentum transfer, $p_{T}^{\min }$. For present analysis the recent version [20] of HYDJET++ is used.

\section{Elliptic, $v_{2}$, and hexadecapole, $v_{4}$, flow}

For our study ca. 60000 minimum bias $\mathrm{Au}+\mathrm{Au}$ collisions at $\sqrt{s}=200 \mathrm{AGeV}$ and ca. 50000 minimum bias $\mathrm{Pb}+\mathrm{Pb}$ collisions at $\sqrt{s}=2.76 \mathrm{ATeV}$ were generated. Figure 1 depicts the excitation functions $v_{2}\left(p_{T}\right)$ and $v_{4}\left(p_{T}\right)$ for the reactions at RHIC and at LHC, respectively. Two centrality bins are selected: (a) $10 \% \leq \sigma / \sigma_{\text {geo }} \leq 20 \%$ and (b) $40 \% \leq \sigma / \sigma_{\text {geo }} \leq 50 \%$. The final distributions are compared to predictions of ideal hydrodynamics and to available experimental data. It is worth mentioning that the saturation and falloff of the elliptic flow in HYDJET++ takes place at $p_{T} \geq 2.5$ $\mathrm{GeV} / c$, whereas in mere hydro-part of the calculations the $v_{2}$ increases instantly. The changing of the regime occurs because at certain transverse momentum jet particles, that provide very weak elliptic flow, start to dominate over the hadrons coming from soft processes, see [22, 23] for details. 

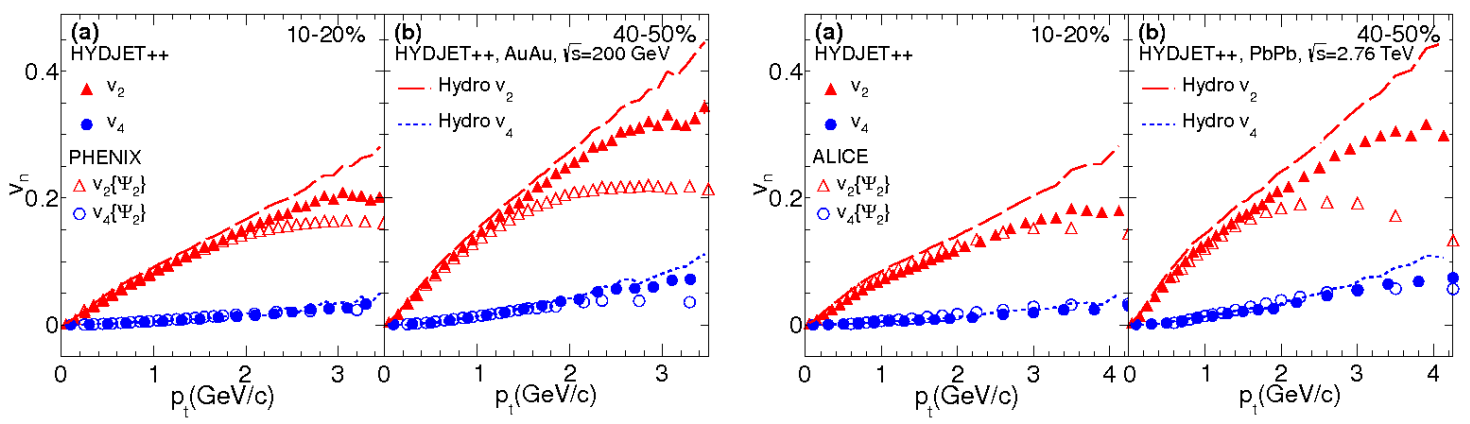

Figure 1: Left plot: $v_{2}\left(p_{T}\right)$ (full triangles) and $v_{4}\left(p_{T}\right)$ (full circles) for charged particles in HYDJET++ calculations of Au+Au collisions at $\sqrt{s}=200 \mathrm{~A} \mathrm{GeV}$ at centrality $\sigma / \sigma_{\text {geo }}$ (a) $10-20 \%$ and (b) $40-50 \%$, respectively. Dashed and dotted lines show hydrodynamic part of the calculations. Data from [8] are shown by open triangles $\left(v_{2}\right)$ and by open circles $\left(v_{4}\right)$. Right plot: The same as left plot but for $\mathrm{Pb}+\mathrm{Pb}$ collisions at $\sqrt{s}=2.76 \mathrm{~A} \mathrm{GeV}$. Data are taken from [21].

As a result, the integrated elliptic flow drops. For semi-central collisions the agreement between the model results and the data is good, whereas for $20 \% \leq \sigma / \sigma_{\text {geo }} \leq 50 \%$ the model overshoots the data at $2 \mathrm{GeV} / c \leq p_{T} \leq 6 \mathrm{GeV} / c$. Then, the $v_{4}$ flow, that originates in the model merely from the $v_{2}$, is weak. Fluctuations in the position of event plane for both elliptic and hexadecapole flow are absent in the HYDJET++. Therefore, (i) the ratio $v_{4} / v_{2}^{2}$ is not changed if one first averages $v_{2}$ and $v_{4}$ over the whole events instead of event-by-event analysis; (ii) we have to compare to that $v_{4}$ flow which was extracted with respect to the event plane angle $\Psi_{2}$ rather than $\Psi_{4}$.

The ratio $R=v_{4} / v_{2}^{2}$ is shown in Fig. 2 for RHIC and in Fig. 3 for LHC. Experimental data plotted onto the obtained results are reduced by factor $1.56(10 \%-20 \%)$ and $1.38(40 \%-50 \%)$ according to analysis of [10]. For the directly produced particles in hydro part of the HYDJET++ the ratio $R$ equals to 0.5 [24]. Feed-down from resonances increases it to 0.6 , and jet contribution brings it to 0.65 (RHIC) and 0.7 (LHC). The most important feature is the rise of $R$ at $p_{T} \geq 2.5 \mathrm{GeV} / c$, seen distinctly at LHC (Fig.3). Hydrodynamics provide almost flat signal, thus it looks like this rise can be attributed solely to jet processes.

\section{NCQ scaling}

The number-of-constituent-quark (NCQ) scaling implies $[12,13]$ the unique scaling trend of the distributions $v_{2}^{*}\left(K E_{T}^{*}\right)$, where $v_{2}^{*}=v_{2} / n_{q}, K E_{T}^{*}=K E_{T} / n_{q}, K E_{T} \equiv m_{T}-m_{0}$ is the transverse kinetic energy, and $n_{q}$ is the number of constituent quarks in a given hadron, i.e. $n_{q}=2$ for mesons and $n_{q}=3$ for baryons. The NCQ scaling at RHIC in observed in a quite broad transverse energy range, $0.2 \mathrm{GeV} \leq K E_{T} \leq 0.7 \mathrm{GeV}$. One of the hypotheses explaining such a behavior was the idea of the formation of elliptic flow already at partonic level [26]. In this case the scaling must be fulfilled in a significantly broader energy range at LHC energies. However, the experiment shows [11] that the scaling holds just approximately within $20 \%$ of accuracy, i.e. it is getting worse compared to 


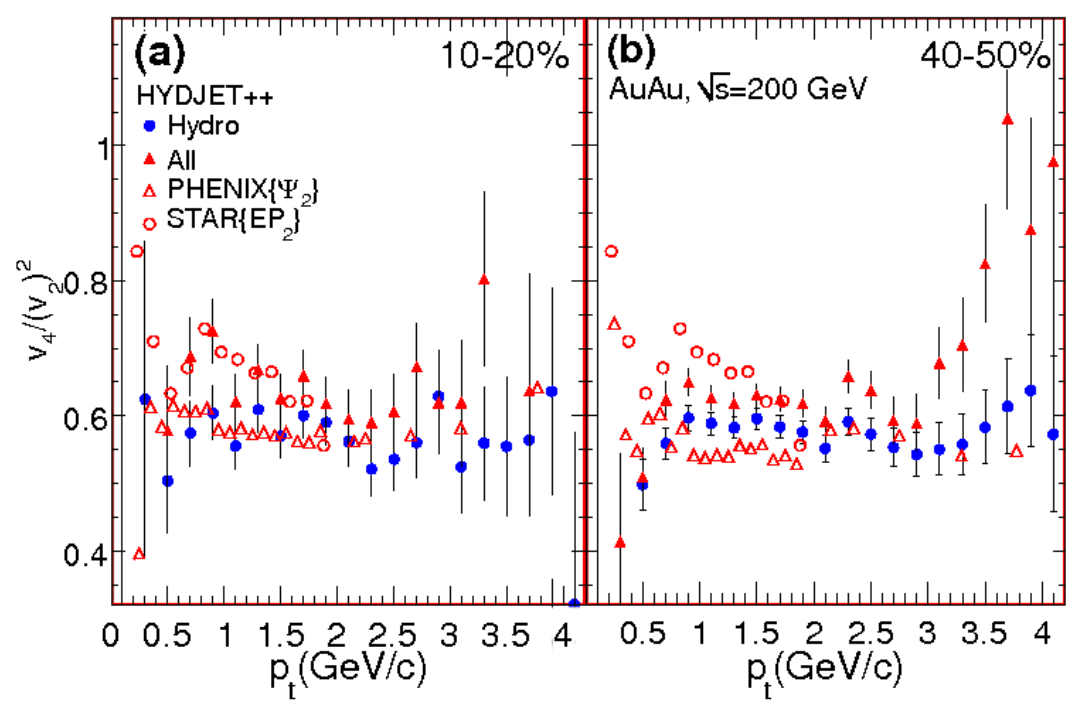

Figure 2: Ratio $v_{4} /\left(v_{2}\right)^{2}$ as a function of $p_{T}$ for charged particles in HYDJET++ hydro (full circles) and hydro+jets (full triangles) calculations of gold-gold collisions at $\sqrt{s}=200 \mathrm{~A} \mathrm{GeV}$ at centrality (a) $10 \%-$ $20 \%$ and $(b) 40 \%-50 \%$. Open circles and open triangles denote data from [7] and [8], respectively.

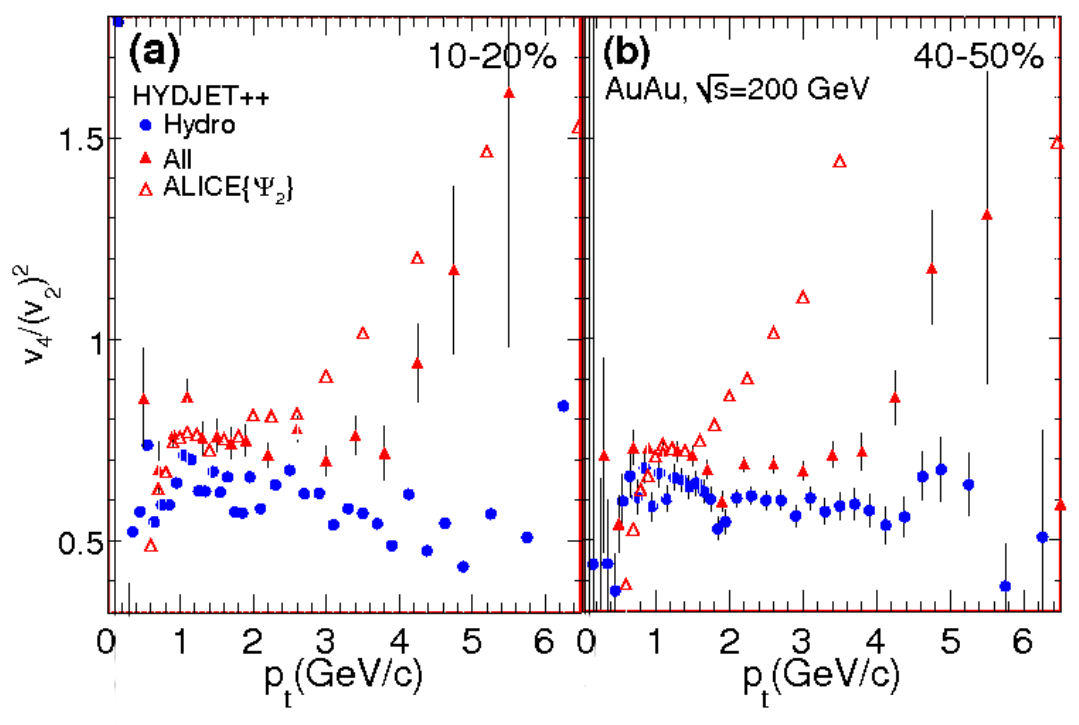

Figure 3: The same as Fig.2 but for $\mathrm{Pb}+\mathrm{Pb}$ collisions at $\sqrt{s}=2.76 \mathrm{~A} \mathrm{GeV}$. Data shown by open triangles are taken from [21]. 

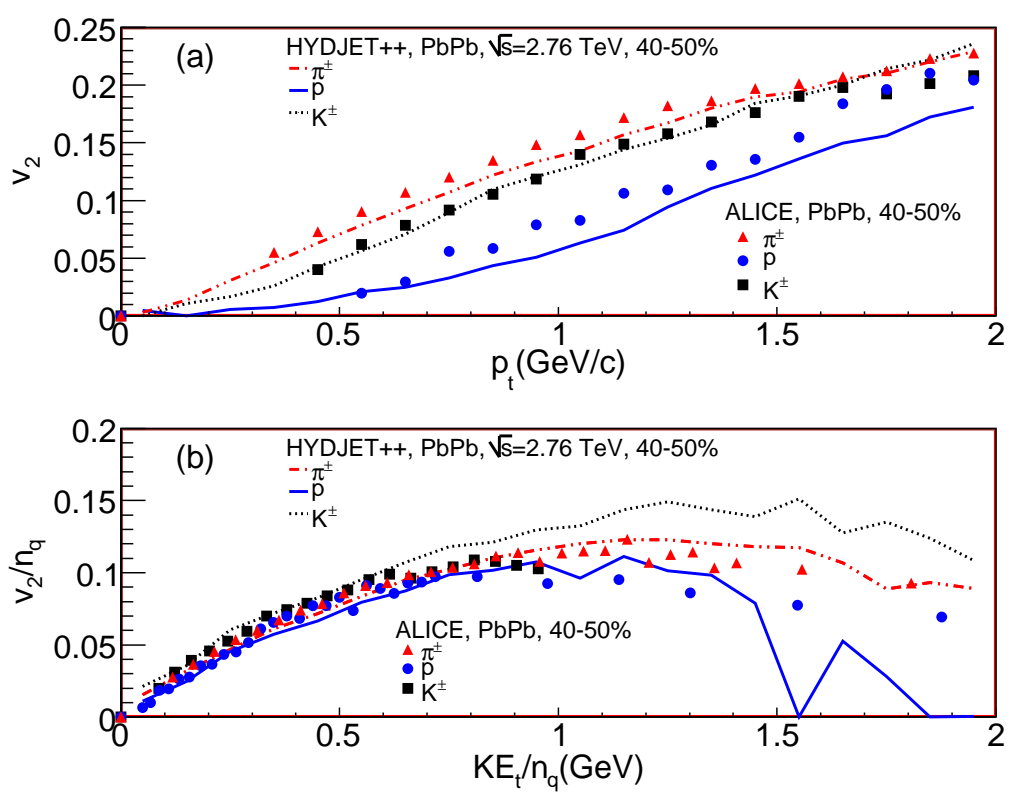

Figure 4: (a) The $p_{T}$-dependence of elliptic flow in HYDJET++ model for different hadron species (lines) in $\mathrm{Pb}+\mathrm{Pb}$ collisions at $\sqrt{s}=2.76 \mathrm{AGeV}$ with centrality $40-50 \%$. Experimental data (symbols) are taken from [25]. (b) The same as (a) but in scaling variables, $v_{2} / n_{q}$ vs. $K E_{T}$.

RHIC. Such a deterioration of the scaling conditions was predicted in $[22,23]$ within the framework of the HYDJET++ model.

Since some tunes of the jet-related part of the model have been changed compared to those used in the previous analysis $[22,23]$, it is necessary to check if the main conclusion of [22] still holds. Figure 4 displays the elliptic flow as a function $p_{T}$ and scaled flow $v_{2} / n_{q}\left(K E_{T} / n_{q}\right)$ for charged pions, kaons and (anti)protons in lead-lead collisions at LHC at centrality $40 \%-50 \%$. It looks like the approximate scaling is fulfilled below $0.7 \mathrm{GeV}$ both in experiment and in the model. However, how approximate is it, and what is the role of jet processes? To answer these questions we plot the $v_{2} / n_{q}$ of the aforementioned particles together with that of lambdas, produced at centrality $20 \%-30 \%$ in $\mathrm{Au}+\mathrm{Au}$ collisions at $\sqrt{s}=200 \mathrm{AGeV}$ and in $\mathrm{Pb}+\mathrm{Pb}$ collisions at $\sqrt{s}=2.76 \mathrm{ATeV}$ in Fig.5 and Fig.6, respectively. Here the flow excitation functions are shown separately for the hadrons produced in hydrodynamic calculations after the resonance feed-down, and for all hadrons produced both in soft and in hard processes. To see the scaling more distinctly these distributions were divided to the flow of protons. The obtained ratios $\left(v_{2}^{(i)} / n_{q}^{(i)}\right) /\left(v_{2}^{p} / 3\right)$ are presented in bottom panels of Figs. 5 and 6. One can see that in the hydrodynamic sector the NCQ-scaling is fulfilled for both reactions. When jet particles are taken into account, then only approximate scaling holds within ca. $15 \%-20 \%$ accuracy limit. Violation of the NCQ-scaling is stronger at LHC compared to RHIC, because the influence of hard processes increases with rising collision energy.

\section{Conclusions}

The following conclusions can be drawn from our study. (i) Despite the weak elliptic and hexadecapole flow of jet hadrons, these particles increase the overall ratio $v_{4} / v_{2}^{2}$ for heavy-ion 


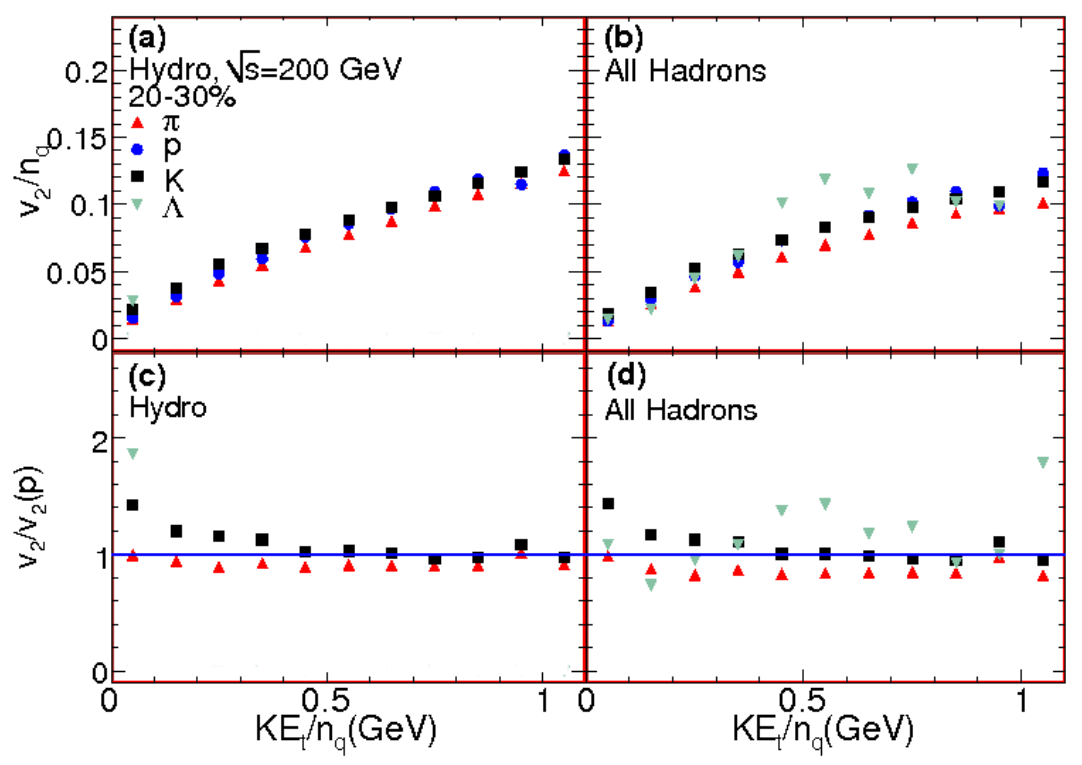

Figure 5: Elliptic flow vs. transverse energy obtained in HYDJET++ for hadrons in (a) hydro+resonances and (b) hydro+resonances+jet calculations of $\mathrm{Au}+\mathrm{Au}$ collisions at $\sqrt{s}=200 \mathrm{AGeV}$ with centrality $20-$ $30 \%$. (c),(d) - the same as (a),(b) but normalized to the flow of protons.

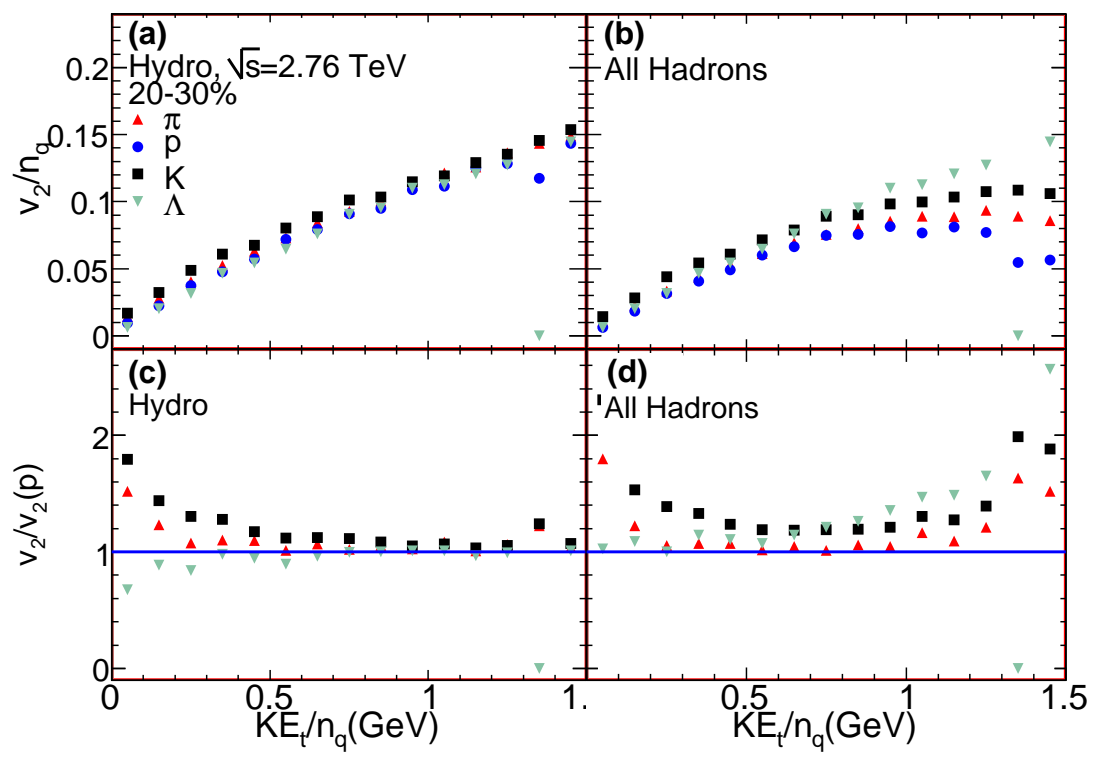

Figure 6: The same as Fig.5 but for $\mathrm{Pb}+\mathrm{Pb}$ collisions at $\sqrt{s}=2.76 \mathrm{~A} \mathrm{GeV}$. 
collisions at ultrarelativistic energies. (ii) Jets can account for the increase of the $v_{4} / v_{2}^{2}$ distribution at $p_{T} \geq 2.5 \mathrm{GeV} / c$, that is absent in both ideal and viscous hydrodynamic model calculations. (iii) Jets are responsible for washing out the NCQ-scaling of elliptic flow of hadrons, although the scaling holds for "hydro-produced" particles.

\section{References}

[1] Y. Schutz and U. Wiedemann (ed.), J. Phys. G 38 issue 12 (Proc. Quark Matter 2011).

[2] E. V. Shuryak, The QCD vacuum, hadrons and superdense matter, World Scientific, Singapore, 2003.

[3] S. A. Voloshin and Y. Zhang, Z. Phys. C 70 (1996) 665.

[4] A. M. Poskanzer and S. A. Voloshin, Phys. Rev. C 58 (1998) 1671.

[5] P. Kolb, Phys. Rev. C 68 (2003) 031902.

[6] N. Borghini and J.-Y. Ollitrault, Phys. Lett. B 642 (2006) 227.

[7] J. Adams et al. (STAR Collaboration), Phys. Rev. Lett. 92 (2004) 062301; Phys. Rev. C 72 (2005) 014904.

[8] A. Adare et al. (PHENIX Collaboration), Phys. Rev. Lett. 105 (2010) 062301.

[9] C. Gombeaud and J.-Y. Ollitrault, Phys. Rev. C 81 (2010) 014901.

[10] M. Luzum, C. Gombeaud, and J.-Y. Ollitrault, Phys. Rev. C 81 (2010) 054901.

[11] M. Krzewicki et al. (ALICE Collaboration), J. Phys. G 38 (2011) 124047.

[12] J. Adams et al. (STAR Collaboration), Phys. Rev. Lett. 92 (2004) 052302.

[13] S. S. Adler et al. (PHENIX Collaboration), Phys. Rev. Lett. 91 (2003) 182301.

[14] I. P. Lokhtin, L. V. Malinina, S. V. Petrushanko, A. M. Snigirev, I. Arsene, and K. Tywoniuk, Comput. Phys. Commun. 180 (2009) 779.

[15] N. S. Amelin et al., Phys. Rev. C 74 (2006) 064901; Phys. Rev. C 77 (2008) 014903.

[16] I. P. Lokhtin and A. M. Snigirev, Eur. Phys. J. C 46 (2006) 211.

[17] G. Torrieri, S. Steinke, W. Broniowski, W. Florkowski, J. Letessier, and J. Rafelski, Comput. Phys. Commun. 167 (2005) 229.

[18] T. Sjostrand, S. Mrenna, and P. Skands, J. High Energy Phys. 0605 (2006) 026; P. Skands, Phys. Rev. D 82 (2010) 074018.

[19] B. Andersson, G. Gustafson, G. Ingelman, and T. Sjostrand, Phys. Rep. 97 (1983) 31.

[20] I. P. Lokhtin, A. V. Belyaev, L. V. Malinina, S. V. Petrushanko, E. P. Rogochaya, and A. M. Snigirev, Eur. Phys. J. C 72 (2012) 2045.

[21] B. Abelev et al. (ALICE Collaboration), arXiv:1205.5761 [nucl-ex].

[22] G. Eyyubova et al., Phys. Rev. C 80 (2009) 064907.

[23] E. Zabrodin et al., J. Phys. G. 37 (2010) 094060.

[24] E. Zabrodin, G. Eyyubova, L. Malinina, and L. Bravina, Acta. Phys. Polon. Supp.5 (2012) 349.

[25] F. Noferini et al. (ALICE Collaboration), arXiv:1212.1292 [nucl-ex].

[26] P. Sorensen, arXiv:0905.0174 [nucl-ex]. 